Copyright 2020 Society of Photo-Optical Instrumentation Engineers. One print or electronic copy may be made for personal use only. Systematic reproduction and distribution, duplication of any material in this paper for a fee or for commercial purposes, or modification of the content of the paper are prohibited. 


\title{
A bistable galloping energy harvester for enhanced concurrent wind and base vibration energy harvesting
}

\author{
Liya Zhao*a

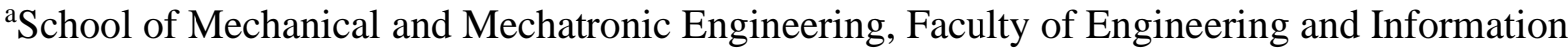 \\ Technology, University of Technology Sydney, 15 Broadway Ultimo 2007, NSW, Australia \\ *liya.zhao@uts.edu.au
}

\begin{abstract}
A compact bistable galloping oscillator is proposed for enhanced power generation from concurrent wind and base vibration. The harvester consists of a D-shaped bluff body attached to a piezoelectric cantilever. Repulsive magnetic interaction is introduced between the bluff body and a fixed windward support to bring nonlinear bistability. An aeroelectro-mechanically coupled model is established and experimentally validated. Both experimental measurements and model predictions demonstrate that the synchronization bandwidth for efficient energy harvesting from concurrent wind and base vibration can be substantially extended.
\end{abstract}

Keywords: Concurrent wind and vibration energy, analytical solution, aeroelasticity, galloping, piezoelectric device.

\section{INTRODUCTION}

The development of distributed low-power microelectronic devices has stimulated growing interests in converting renewable kinetic energy into electrical power to enable perpetually self-powered systems. Researchers have proposed design and efficiency enhancement methods for harnessing energy from pre-existing base vibrations [1-4,24,25]. Others have reported to harness energy from wind by exploring aeroelastic instabilities, such as galloping, flutter, vortex-induced vibration (VIV), etc [5-15]. Indeed, these two types of kinetic energy sources, i.e., base vibration and wind energy, are coexisting in many environments, like ocean buoys, bridges and vehicles. Recently, recognizing that separately charactering the dynamic responses of a single source type is inadequate because of their coupling and interaction, researchers have started to study the characteristics of energy harvesting under concurrent loadings [16-19]. Bibo and Daqaq [16] studied the performance of a flutter energy harvester under concurrent wind and base vibratory excitations. Enhanced power was obtained when base excitation frequencies are very close to the harvester's resonance. However, if the frequency is slightly away, the power level is limited to that resulting from flutter alone. Yan et al. [18] numerically analyzed the influences of the wind speed, base acceleration and load resistance on the dual frequency response of a linear galloping harvester with a triangular sectioned bluff body. Dai et al. [19] numerically studied the concurrent wind and base vibration energy harvesting performance of a VIV energy harvester with a circular cylinder at the tip. In both studies, however, effective concurrent energy conversion is limited to a narrow frequency range. To enhance concurrent wind and base vibration energy harvesting and broaden the frequency bandwidth with synchronized aeroelastic and base excited oscillations, Zhao and Yang [20] proposed an impact-based galloping energy harvester with a mechanical stopper and experimentally validated its capability of generating enhanced power over a significantly extended bandwidth. Zhao [21,22] further analyzed the potential advantage of utilizing the high frequency mechanical stopper as a complementary harvester, and derived the close-form analytical solutions for power prediction. Employing the 3:1 internal resonance, Liu and Gao [23] theoretically studied enhanced power responses of a flutter energy harvester under combined loadings.

In this paper, a compact broadband galloping energy harvester for enhanced concurrent wind and base vibration energy harvesting is proposed, as shown in Fig. 1. The harvester consists of a bluff body prism that is rigidly attached to the free end of a cantilever. To convert the structural bending strain energy into electrical power, a piezoelectric transducer is glued onto the surface of the cantilever. The bluff body has a D-shape cross section. When the wind is flowing toward its flat surface, large-amplitude galloping oscillations arise as a result of unbalanced pressures in the top and bottom shear layers. The harvester system is also subjected to base vibratory excitations. Two repulsive magnets are attached on the bluff body 
and a windward support to introduce bistable stiffness nonlinearity [24,25]. The structural bistability significantly extends the synchronization bandwidth for effective concurrent energy harvesting within which the two energy sources are simultaneously harnessed by locking-in and supplementing each other.

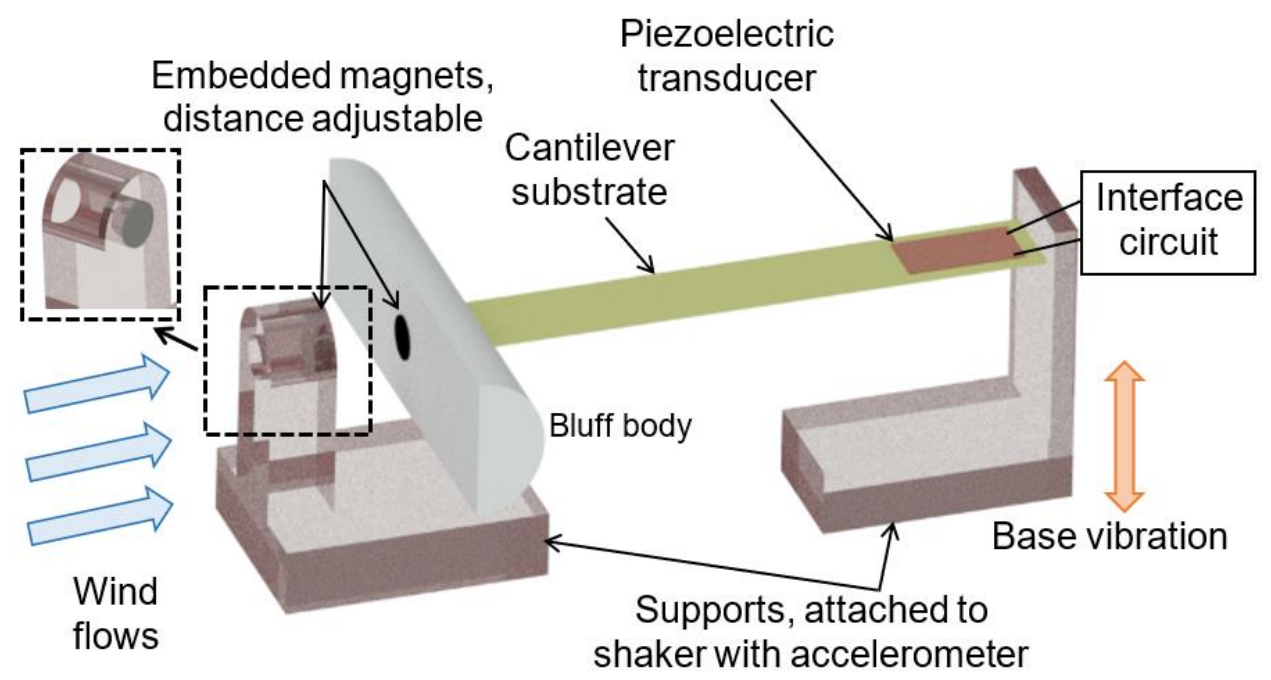

Figure 1. Schematic of the bistable galloping energy harvesting for broadband concurrent wind and base vibration energy harvesting

\section{MATHEMATICAL MODELING}

The analytical model for the proposed concurrent wind and base vibration energy harvester with aero-electro-mechanical coupling is expressed as

$$
\left\{\begin{array}{l}
M_{0} \ddot{u}+C_{0} \dot{u}+K_{0} u+F_{m}+\Theta \bar{V}=F_{\text {galloping }}-M_{0} \ddot{z}_{0} \\
I+C_{p} \dot{V}-\Theta \dot{u}=0
\end{array}\right.
$$

where $u$ is the relative bluff body displacement to the base, $z_{0}$ is the base excitation, $V$ and $I$ are the voltage and current outputs across the piezoelectric laminate, $M_{0}, C_{0}, K_{0}, C_{p}$ and $\Theta$ are, respectively, the effective mass, damping, stiffness of the initial linear structure, piezoelectric capacitance and electromechanical coupling. $F_{m}$ is the nonlinear restoring force induced by the repulsive magnets, which can be experimentally obtained. The aerodynamic model to represent the galloping force $F_{\text {galloping }}$ is built based on the quasi-steady hypothesis [26], given by

$$
F_{\text {galloping }}=\frac{1}{2} \rho h L U^{2}\left[\sum_{i=1}^{3} A_{i}\left(\frac{\dot{u}+\dot{z}_{0}}{\bar{U}}+\lambda u\right)^{i}\right]-M_{0} \ddot{z}_{0}
$$

where $U$ is the wind speed, and $\rho, h, L$ and $A_{i}$ denote, respectively, the air density, cross-flow diameter and length of the bluff body prism, and empirical aerodynamic coefficients. In Eqs. (1) and (2), the overdot represents the derivative with respect to time.

\section{EXPERIMENTAL VALIDATION}

To verify the wideband synchronization characteristics of the proposed harvester, experiment is conducted with a fabricated prototype. The cantilever is made of aluminium, with a D-shape foam bluff body attached at the free end. A neodymium magnet is attached to the bluff body. Another neodymium magnet is fixed to a support in front of the bluff body, which is repulsive to the magnet on the bluff body. An MFC (Smart Materials Corp.) piezoelectric sheet is bonded onto the cantilever near the clamped end. Across the electrodes the energy harvesting circuit is connected. In this study, a simple ac circuit consisting of a pure resistive load $R$ is employed to evaluate the strain-to-electricity conversion, while the ac-dc conversion, power regulation or storage are not considered [27-29]. In Eq. (1), $I$ is related to $V$ by $I=V / R$. 
The system is mounted onto an electromagnetic shaker which provides base excitations. The acceleration is monitored by an accelerometer. A fan is placed in front of the prototype to generate wind flows. The wind speed is measured by an anemometer. The NI 9229 data acquisition system is used to measure the voltage. The system parameters are identified through the equivalent lump parameter transformation method as detailed in our previous studies [20,27], as shown in Table 1. The aerodynamic coefficients and nonlinear stiffness terms $\left(F_{m}=-\alpha u+\beta u^{3}\right)$ are obtained by fitting predictions against experimental measurements under galloping alone and base excitation alone conditions.

Table 1. Identified system parameters

\begin{tabular}{|c|c|c|c|c|}
\hline$M(\mathrm{~g})$ & $\mathrm{C}\left(\mathrm{Nsm}^{-1}\right)$ & $\mathrm{K}_{0}\left(\mathrm{Nm}^{-1}\right)$ & $\Theta\left(\mathrm{NV}^{-1}\right)$ & $C_{p}(\mathrm{nF})$ \\
\hline 5.82 & 0.0091 & 35.774 & $8.49 \times 10^{-5}$ & 25.7 \\
\hline$A_{1}$ & $A_{2}$ & $A_{3}$ & $\alpha\left(\mathrm{Nm}^{-1}\right)$ & $\beta\left(\mathrm{Nm}^{-3}\right)$ \\
\hline 1.56 & 0 & -6.9 & 57.238 & $2.096 \times 10^{5}$ \\
\hline
\end{tabular}

The predicted and measured root-mean-square (RMS) voltage responses over a range of base excitation frequency are depicted in Figures 2 and 3. The wind and base vibration conditions are constant at $U=4.7 \mathrm{~m} / \mathrm{s}$ and RMS $\ddot{z}_{0}=1 \mathrm{~m} / \mathrm{s}^{2}$. The base excitation frequency is swept up. It is seen from Figure 2 that for the linear galloping harvester, only within a narrow frequency range close to the resonance, high voltage output is achieved. In this range, the galloping frequency is synchronized with the base excitation frequency, making the oscillation of the harvester periodic. Outside of this synchronization region, the oscillation is quasi-periodic with an amplitude modulation due to the two concurrent excitation frequencies. With such quasi-periodic responses, the voltage is almost equal to that from pure galloping, which implies that base vibration energy is not effectively harnessed. In contrast, the bistable galloping harvester achieves a greatly widened synchronization region with periodic oscillations and high-level voltage outputs, where both wind and base vibration energy are truly concurrently harnessed. In the experiment, large-amplitude inter-well oscillations are observed in this region. A synchronization region from $8.5 \mathrm{~Hz}$ to $12 \mathrm{~Hz}$ is achieved with higher voltage output than that from pure galloping. The correlation between the model predictions and experimental measurements is reasonably good. The trend is well approximated. The discrepancies in the amplitude could be attributed to the wind turbulences from fan, the damping changes with the variation of frequency and the cubic polynomial approximation of the magnetic force.

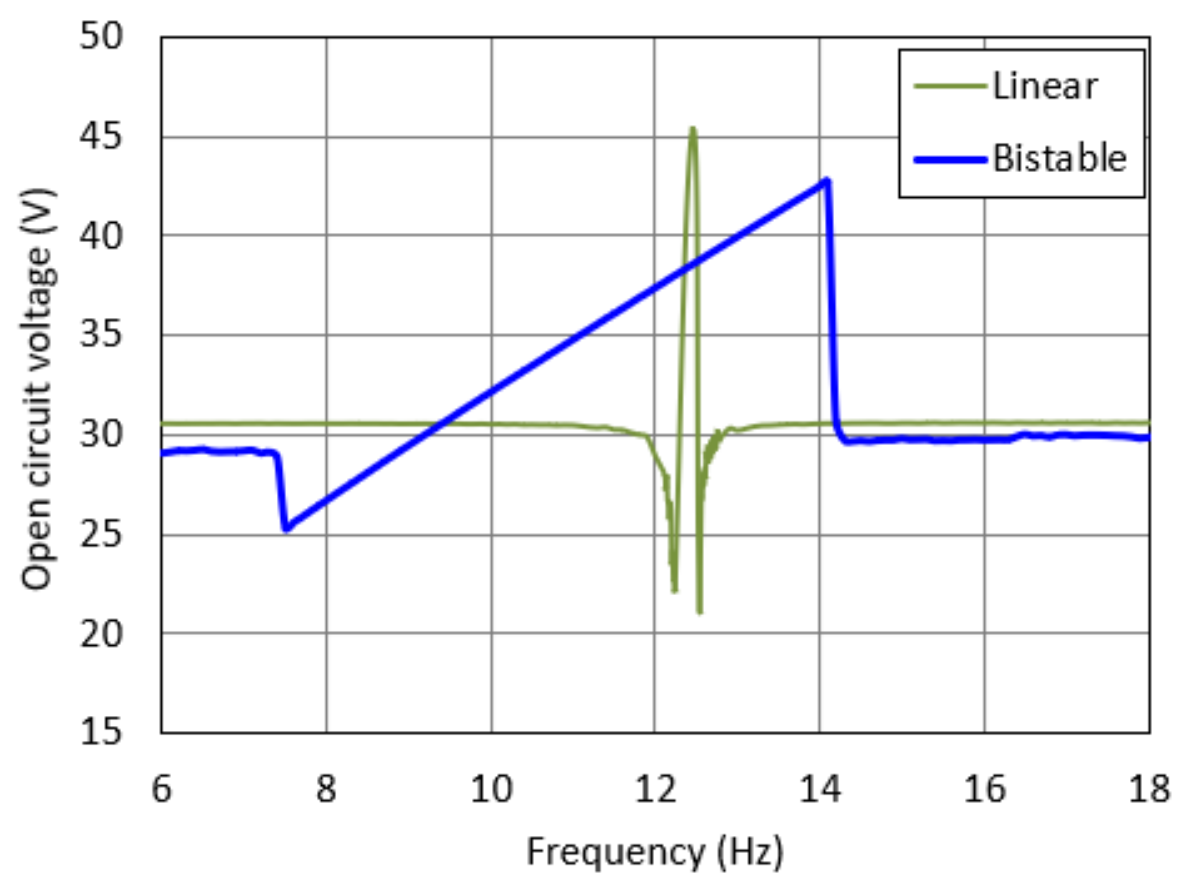

Figure 2. Predicted voltage variation with base excitation frequency with wind speed $U=4.7 \mathrm{~m} / \mathrm{s}$ and RMS acceleration $\ddot{z}_{0}=1 \mathrm{~m} / \mathrm{s}^{2}$. 


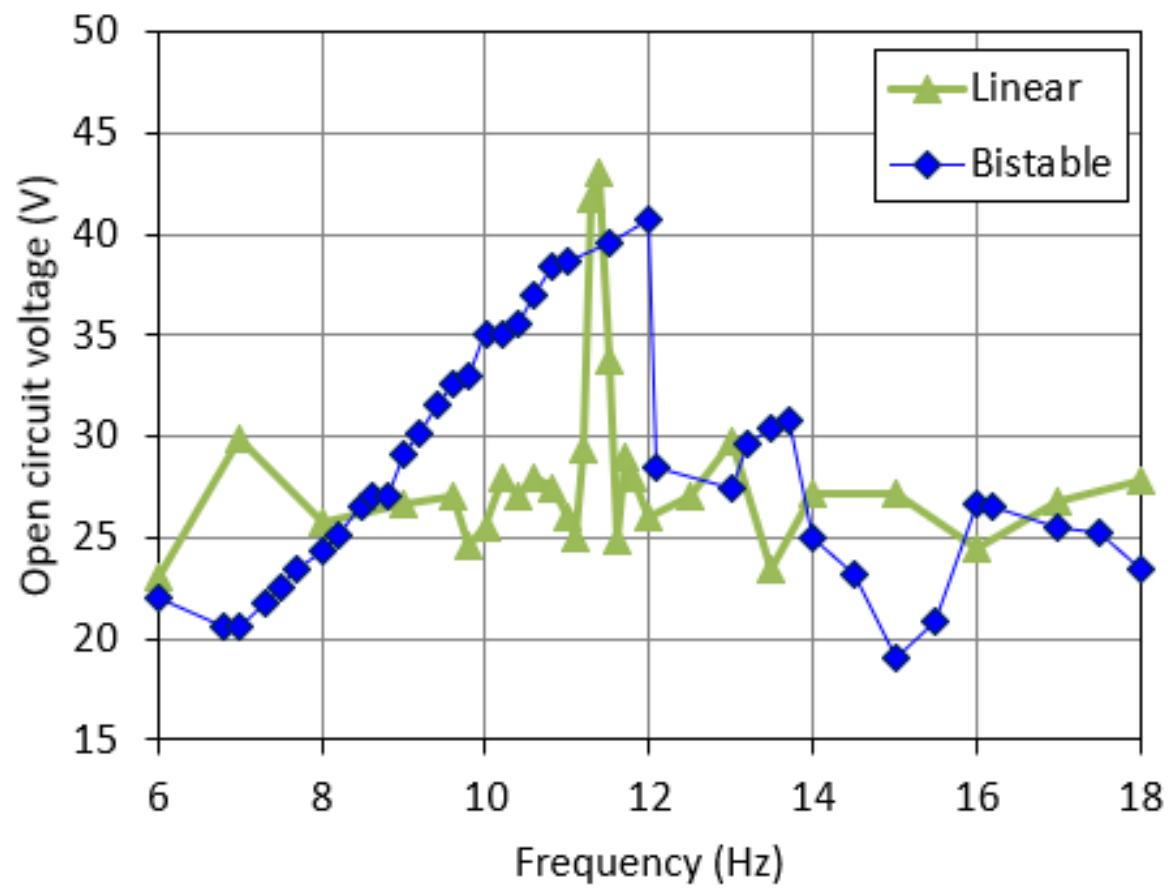

Figure 3. Experimenal voltage variation with base excitation frequency with wind speed $U=4.7 \mathrm{~m} / \mathrm{s}$ and RMS acceleration $\ddot{z}_{0}=1 \mathrm{~m} / \mathrm{s}^{2}$.

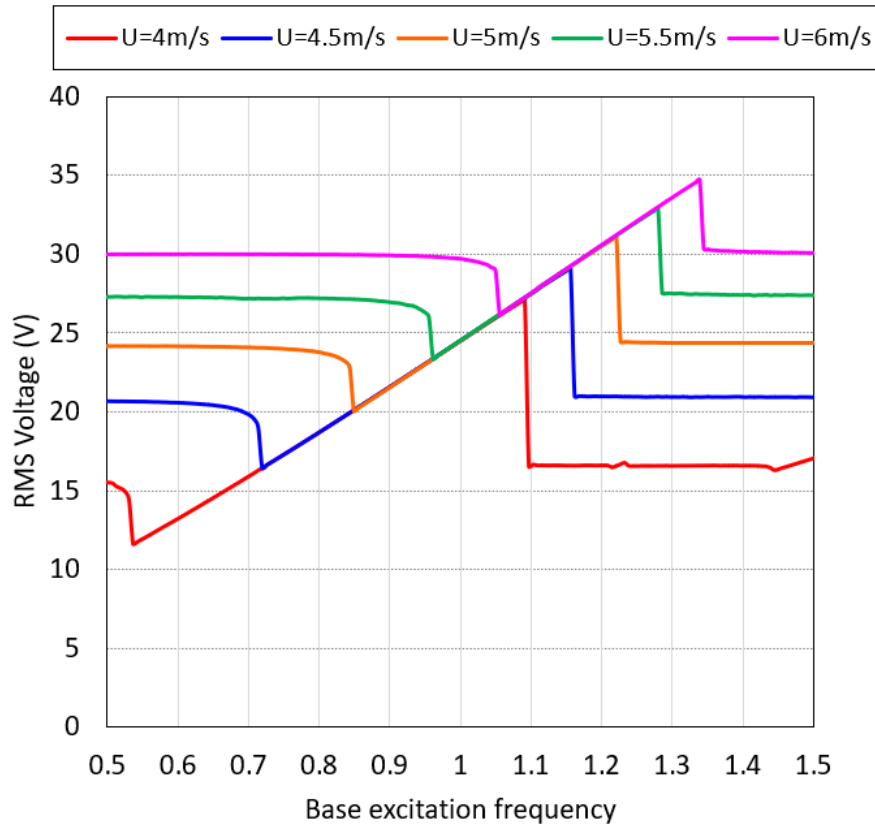

Figure 4. Variation of RMS voltage with base excitation frequency at different wind speeds

\section{PERFORMANCE STUDY}

In this section, the effects of wind speed $U$ and load resistance $R$ on the performance of the harvester are investigated. The parameters employed in the calculations are $M_{0}=2.783 \mathrm{~g}, C_{0}=0.0065 \mathrm{Nsm}^{-1}, K_{0}=31.56 \mathrm{Nm}^{-1}, \Theta=9.60 \times 10^{-5} \mathrm{NV}^{-1}, C_{p}=25.7 \mathrm{nF}$, $\left[A_{1} A_{2} A_{3}\right]=[2.30-18], \alpha=47.34 \mathrm{Nm}^{-1}$ and $\beta=3.156 \times 10^{5} \mathrm{Nm}^{-3}$. The variation of the RMS voltage with the dimensionless base 
excitation frequency $\Omega_{b}$ at different wind speeds $U$ are plotted in Figure 4, where $\Omega_{b}=\omega_{b} / \omega_{n}$ with $\omega_{b}$ and $\omega_{n}$ being the base excitation frequency and the initial linear harvester's fundamental frequency, respectively. The results are based on a constant load resistance $R$ of $333 \mathrm{k} \Omega$. At all considered wind speeds, galloping is fully excited to give large-orbit interwell oscillations. An upsweep $\Omega_{b}$ is conducted. It shows that the voltage amplitude increases with increasing $U$ both inside and outside of the synchronization bandwidth. Moreover, the bandwidth is shifted to higher frequency range at higher $U$. Inspecting the voltage variation, it is seen that increasing $U$ results in a decease in the size of the synchronization bandwidth. The voltage enhancement compared with that of pure galloping excitation is also decreased with increasing $U$. However, since at a large wind speed, galloping alone can already produce a substantially high voltage, the relative disadvantage of a small bandwidth is alleviated.

Figure 5 shows the variation of the average power $P_{a v e}=V_{R M S^{2}} / R$ with the load resistance $R$ at different wind speeds $U$. The results are based on a base excitation frequency to the left of the synchronization bandwidth in Figure 5(a), inside the synchronization bandwidth in Figure 5(b) and to the right of the bandwidth (Figure 5(c). A small initial displacement disturbance of $1 \mathrm{~mm}$ is applied for all the plots. Outside of the bandwidth, it is seen in both Figures 5(a) $\left(\Omega_{b}=0.47\right)$ and 5 (c) $\left(\Omega_{b}=1.36\right)$ that at each wind speed, a single optimal $R$ is present that gives the highest power. Moreover, at each $U$, the optimal R does not change with $\Omega_{b}$ but remains the same for $\Omega_{b}=0.47$ and $\Omega_{b}=1.36$. The presence of only one instead of two optimal $R$ as well as its invariance reveal that the response is dominant at the galloping frequency, while the involvement of base excitation induced response component is negligible. With increasing wind speed, the slight decrease in the optimal $R$ should be due to the slight change in the galloping frequency. Since the bandwidth shifts with the wind speed, in Figure 5(b), $\Omega_{b}$ is not constant but in the middle of the bandwidth for respective $U$. It is seen that at smaller $U$ of $4 \mathrm{~m} / \mathrm{s}$ and $4.5 \mathrm{~m} / \mathrm{s}$, the powers drop in the middle ranges of $R$. The synchronization feature is lost in these ranges, where the time-domain responses revert to quasi-periodic oscillations. It is revealed that the change of $R$ and the associated change of electrical damping resulting from the electromechanical coupling have a strong influence on the response frequency synchronization phenomenon, which deserves further investigations.

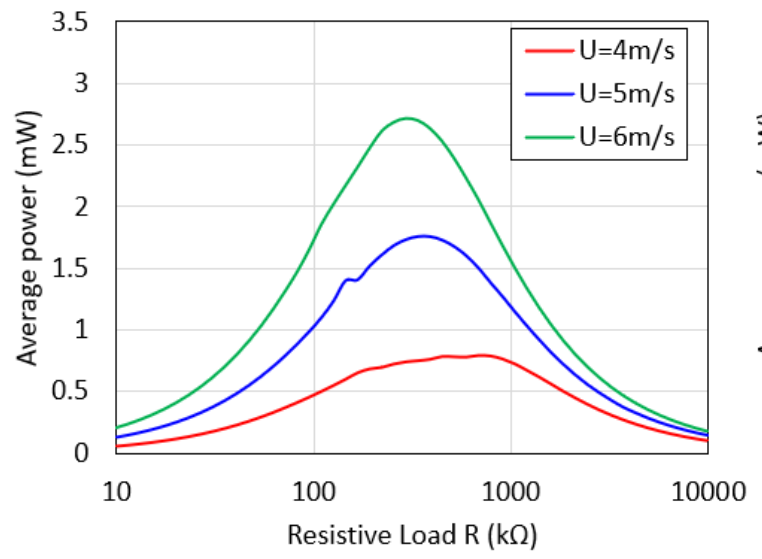

(a)

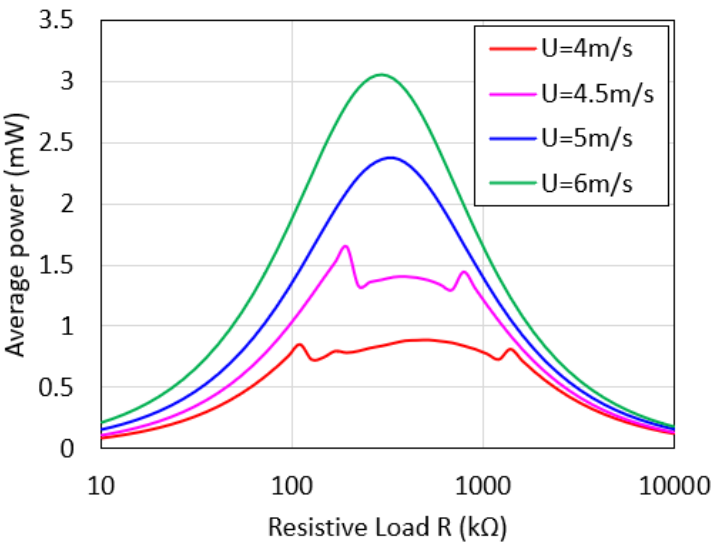

(b)

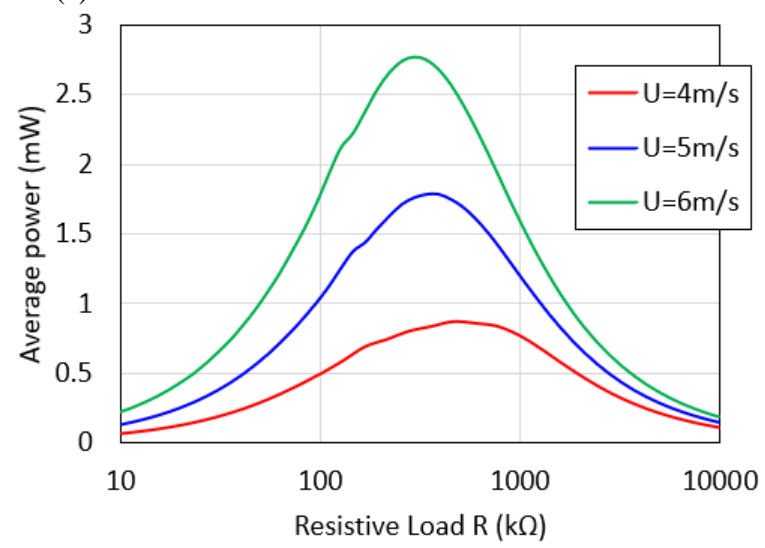

(c)

Figure 5. Variation of average power with load resistance at different wind speeds. Base excitation frequency is (a) outside, to the left (b) inside and (c) outside, to the right of the synchronization bandwidth. 


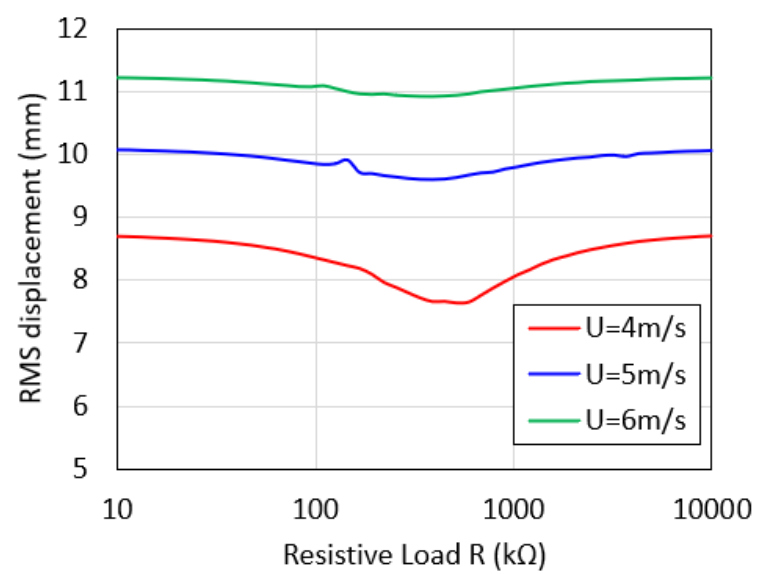

(a)

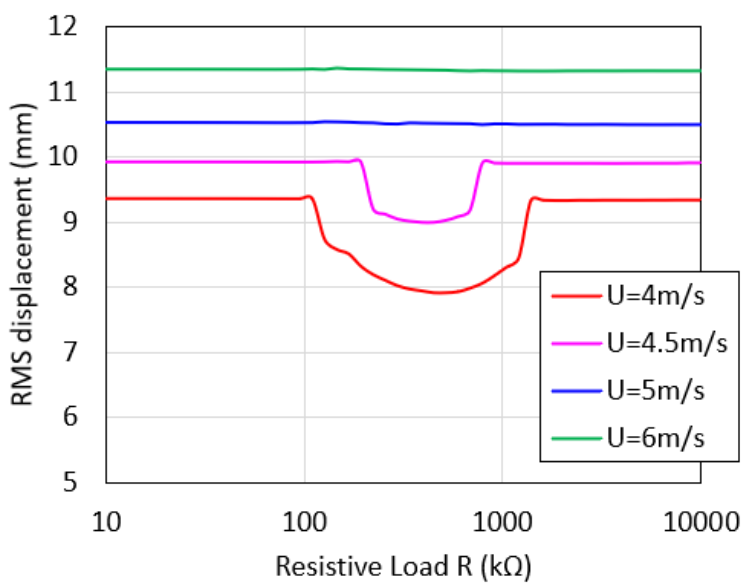

(b)

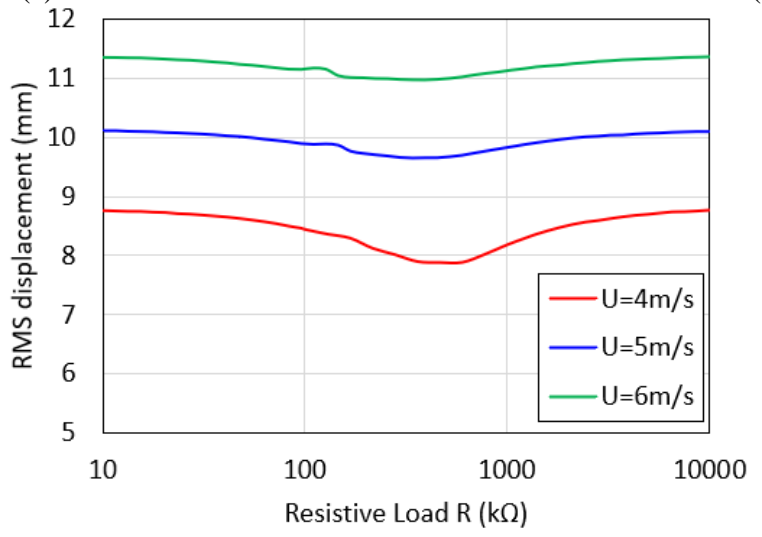

(c)

Figure 6. Variation of RMS displacement with load resistance at different wind speeds. Base excitation frequency is (a) outside, to the left (b) inside and (c) outside, to the right of the synchronization bandwidth.

The variations of RMS displacement with the load resistance at different wind speeds are given in Figure 6, for base excitation frequencies outside (Figures 6(a) and 6(c)) and inside of the synchronization bandwidth (Figure 6(b)). It is seen that outside of the bandwidth, there is a valley in the displacement response at the corresponding optimal $R$ which gives the largest electrical damping. However, it is seen in Figure 6(b) that, inside the synchronization bandwidth, the RMS displacement is almost constant over the whole range of $R$ for $U=5 \mathrm{~m} / \mathrm{s}$ and $6 \mathrm{~m} / \mathrm{s}$ and in most ranges of $R$ for $U=4 \mathrm{~m} / \mathrm{s}$ and $4.5 \mathrm{~m} / \mathrm{s}$. It reflects that as long as synchronization occurs, the oscillation amplitude will be independent of coupling induced electrical damping, which is significantly different from the behavior of energy harvesting under a single excitation source, either aeroelastic galloping or pure base vibration.

\section{CONCLUSION}

In this paper, a compact bistable galloping oscillator is studied for enhanced power generation from concurrent wind and base vibration. An aero-electro-mechanically coupled model is established. The model is validated by experiment with a fabricated prototype. Both experimental measurements and model predictions show that introduction of stiffness bistability can effectively widen the synchronization bandwidth in concurrent wind and base vibration energy harvesting. 


\section{REFERENCES}

[1] K. Fan, Q. Tan, Y. Zhang, S. Liu, M. Cai, and Y. Zhu, "A monostable piezoelectric energy harvester for broadband low-level excitations," Applied Physics Letters, vol. 112, no. 12, p. 123901, 2018.

[2] Y. Shu, W. Wang, and Y. Chang, "Electrically rectified piezoelectric energy harvesting induced by rotary magnetic plucking," Smart Materials and Structures, vol. 27, no. 12, p. 125006, 2018.

[3] F. Gao, G. Liu, B. L.-H. Chung, H. H.-T. Chan, and W.-H. Liao, "Macro fiber composite-based energy harvester for human knee," Applied Physics Letters, vol. 115, no. 3, p. 033901, 2019.

[4] S. Zhou, J. Cao, A. Erturk, and J. Lin, "Enhanced broadband piezoelectric energy harvesting using rotatable magnets," Applied Physics Letters, vol. 102, no. 17, p. 173901, 2013.

[5] C. Kitio Kwuimy, G. Litak, M. Borowiec, and C. Nataraj, "Performance of a piezoelectric energy harvester driven by air flow," Applied Physics Letters, vol. 100, no. 2, p. 024103, 2012.

[6] J. Sirohi and R. Mahadik, "Harvesting Wind Energy Using a Galloping Piezoelectric Beam," Journal of Vibration and Acoustics, vol. 134, no. 1, p. 011009, 2012.

[7] L. Zhao and Y. Yang, "Enhanced aeroelastic energy harvesting with a beam stiffener," Smart Materials and Structures, vol. 24, no. 3, p. 032001, 2015.

[8] J. A. d. C. Dias, C. De Marqui Jr, and A. Erturk, "Hybrid piezoelectric-inductive flow energy harvesting and dimensionless electroaeroelastic analysis for scaling," Applied Physics Letters, vol. 102, no. 4, p. 044101, 2013.

[9] G. Hu, K.-T. Tse, K. C. Kwok, J. Song, and Y. Lyu, "Aerodynamic modification to a circular cylinder to enhance the piezoelectric wind energy harvesting," Applied Physics Letters, vol. 109, no. 19, p. 193902, 2016.

[10] G. Hu, K. Tse, M. Wei, R. Naseer, A. Abdelkefi, and K. Kwok, "Experimental investigation on the efficiency of circular cylinder-based wind energy harvester with different rod-shaped attachments," Applied energy, vol. 226, pp. 682-689, 2018.

[11]P. Chatterjee and M. Bryant, "Analysis of tension-tunable clamped-clamped piezoelectric beams for harvesting energy from wind and vibration," Journal of Intelligent Material Systems and Structures, vol. 30, no. 16, pp. 24052420, 2019.

[12] J. Wang, W. Zhao, Z. Su, G. Zhang, P. Li, and D. Yurchenko, "Enhancing vortex-induced vibrations of a cylinder with rod attachments for hydrokinetic power generation," Mechanical Systems and Signal Processing, vol. 145, p. $106912,2020$.

[13] J. Wang, Z. Su, H. Li, L. Ding, H. Zhu, and O. Gaidai, "Imposing a wake effect to improve clean marine energy harvesting by flow-induced vibrations," Ocean Engineering, vol. 208, p. 107455, 2020.

[14] J. Wang, L. Geng, L. Ding, H. Zhu, and D. Yurchenko, "The state-of-the-art review on energy harvesting from flowinduced vibrations," Applied Energy, vol. 267, p. 114902, 2020.

[15] G. Hu, J. Wang, Z. Su, G. Li, H. Peng, and K. Kwok, "Performance evaluation of twin piezoelectric wind energy harvesters under mutual interference," Applied Physics Letters, vol. 115, no. 7, p. 073901, 2019.

[16] A. Bibo and M. F. Daqaq, "Investigation of concurrent energy harvesting from ambient vibrations and wind using a single piezoelectric generator," Applied Physics Letters, vol. 102, no. 24, p. 243904, 2013.

[17] A. Bibo and M. F. Daqaq, "An analytical framework for the design and comparative analysis of galloping energy harvesters under quasi-steady aerodynamics," Smart Materials and Structures, vol. 24, no. 9, p. 094006, 2015.

[18]Z. Yan, A. Abdelkefi, and M. R. Hajj, "Piezoelectric energy harvesting from hybrid vibrations," Smart Materials and Structures, vol. 23, no. 2, p. 025026, 2014.

[19]H. L. Dai, A. Abdelkefi, and L. Wang, "Piezoelectric energy harvesting from concurrent vortex-induced vibrations and base excitations," Nonlinear Dynamics, vol. 77, no. 3, pp. 967-981, 2014.

[20]L. Zhao and Y. Yang, "An impact-based broadband aeroelastic energy harvester for concurrent wind and base vibration energy harvesting," Applied Energy, vol. 212, pp. 233-243, 2018.

[21]L. Zhao, "Performance Enhancement of an Aeroelastic Energy Harvester for Efficient Power Harvesting from Concurrent Wind Flows and Base Vibrations," presented at the IEEE/ASME AIM, 2018.

[22] L. Zhao, "Analytical solutions for a broadband concurrent aeroelastic and base vibratory energy harvester," presented at the SPIE Active and Passive Smart Structures and Integrated Systems XII, 2019.

[23] H. Liu and X. Gao, "Vibration energy harvesting under concurrent base and flow excitations with internal resonance," Nonlinear Dynamics, vol. 96, no. 2, pp. 1067-1081, 2019.

[24]R. Harne and K. Wang, "A review of the recent research on vibration energy harvesting via bistable systems," Smart Materials and Structures, vol. 22, no. 2, p. 023001, 2013. 
[25] M. F. Daqaq, R. Masana, A. Erturk, and D. D. Quinn, "On the role of nonlinearities in vibratory energy harvesting: a critical review and discussion," Applied Mechanics Reviews, vol. 66, no. 4, p. 040801, 2014.

[26] M. P. Païdoussis, S. J. Price, and E. De Langre, Fluid-structure interactions: Cross-flow-induced instabilities. New York: Cambridge University Press, 2010.

[27]L. Zhao and Y. Yang, "Analytical solutions for galloping-based piezoelectric energy harvesters with various interfacing circuits," Smart Materials and Structures, vol. 24, no. 7, p. 075023, 2015.

[28]L. Zhao, L. Tang, J. Liang, and Y. Yang, "Synergy of wind energy harvesting and synchronized switch harvesting interface circuit," IEEE/ASME Transactions on Mechatronics, vol. 22, no. 2, pp. 1093-1103, 2016.

[29] L. Zhao and Y. Yang, "Comparison of four electrical interfacing circuits in wind energy harvesting," Sensors and Actuators A: Physical, vol. 261, pp. 117-129, 2017. 